\title{
Thymic neuroendocrine tumor in a patient with multiple endocrine neoplasia type I : The limitations of surveillance
}

\author{
Kimberly C Zibert, Curtis J Hobbs \\ Madigan Army Medical Center, Tacoma, United States. \\ Correspondence: Kimberly C Zibert. Address: Madigan Army Medical Center, Tacoma, United States. \\ Email: kimberly.c.zibert.mil@mail.mil \\ Received: August 30, 2014 \\ Accepted: September 20, 2014 Online Published: November 12, 2014 \\ DOI : $10.5430 /$ crim.v2n1p73 \\ URL: http://dx.doi.org/10.5430/crim.v2n1p73
}

\begin{abstract}
Multiple endocrine neoplasia type I (MEN1) is an autosomal dominant disorder associated with pituitary and parathyroid adenomas and neuroendocrine tumors (NETs) of the pancreas. Pancreatic NETs, most of which are non-functional, are now the primary life-threatening manifestation of MEN1. Some patients with this disorder develop NETs of the thymus, which are typically characterized by aggressive behavior and malignant potential. Although rare in the general population, $25 \%$ of all reported thymic NETs have occurred in patients with MEN1. With the aid of advancements in imaging and biomarkers, the clinician is now better equipped in detecting NETs in this high-risk group. In this report, we present a patient whose incidentally discovered thymic NET led to the diagnosis of MEN1. We provide an overview of MEN1associated NETs with a specific focus on the use of chromogranin A (CgA) in disease surveillance.
\end{abstract}

\section{Keywords}

Multiple endocrine neoplasia type I, MEN1, Neuroendocrine tumors, Chromogranin A

\section{I ntroduction}

Multiple endocrine neoplasia type I is an autosomal dominant disorder causing tumors of the parathyroid, pituitary, and pancreas. Parathyroid adenomas are the most common manifestation of MEN1 followed by neuroendocrine tumors (NETs) of the pancreas, with a prevalence of $95 \%$ and $70 \%$, respectively ${ }^{[1]}$. Pancreatic NETs include functional gastrinomas, insulinomas, glucagonomas, and vasoactive intestinal polypeptidomas (VIPomas), as well as nonfunctioning tumors. In addition, patients with MEN1 are more likely to develop pituitary adenomas, benign adrenal tumors, skin tumors (angiofibromas, collangenomas and lipomas), and NETs of the thymus, stomach, and bronchus ${ }^{[2,3]}$. We present a patient whose thymic NET led to the diagnosis of MEN1. We discuss thymic and pancreatic NETs, summarize current MEN1 surveillance guidelines, and discuss the role of chromogranin A (CgA) in NET surveillance.

\section{Case presentation}

Mr. P.B. was a 53-year-old Caucasian man with past medical history of diabetes, hypertension, acid reflux disease, pulmonary nodules, and primary hyperparathyroidism. His surgical history was significant for parathyroidectomy, which 
was done when he was in his early forties. He also reported excision of an abdominal lipoma. His medications included several antihypertensives, an oral hypoglycemic agent, and a twice daily proton pump inhibitor (PPI). He reported drinking 1-2 beers on the weekend. He denied illicit drug use. He reported a remote history of both smokeless tobacco and cigarette use. His family history was significant for a mother with parathyroid and pancreatic tumors, and a maternal grandfather and maternal uncle both with parathyroid adenomas. His mother had declined genetic screening for MEN1. He has no siblings and no children.

Five years prior to the diagnosis of his thymic neuroendocrine tumor (NET), our patient had a computed tomography (CT) of the chest that disclosed multiple pulmonary nodules and enlarged mediastinal lymph nodes. At the time these findings were attributed to a resolving upper respiratory infection. He was encouraged to have a follow-up chest CT in three months, but he failed to comply.

At the time his thymic NET was diagnosed, the chest CT was demonstrating a $3.7 \mathrm{~cm}$ mediastinal mass, a $1.6 \mathrm{~cm}$ pre-vascular lymph node, and multiple pulmonary nodules. Our patient denied constitutional symptoms, weight loss, chest pain, or dyspnea but reported a chronic productive cough and nasal drainage. He was referred to a cardiothoracic surgeon and underwent a median sternotomy with en-bloc excision of the thymus and two adjacent lymph nodes. Pathology identified a well-differentiated thymic NET with clear margins. Both lymph nodes demonstrated evidence of vascular invasion. A somatostatin receptor scintigraphy scan (SRS) was negative for residual or metastatic disease. Neither radiation nor chemotherapy was recommended. Genetic testing revealed a mutation in the menin gene, resulting in a heterozygous nucleic acid change: c.1251C > G (p.Tyr417Stop). Mr. P.B. was referred to the Endocrinology Clinic for further MEN1 work up and surveillance.

\section{Discussion}

MEN1 syndrome is an autosomal dominant disorder resulting from a genetic mutation in the MEN1 tumor suppressor gene characteristically resulting in parathyroid, pituitary, and pancreatic tumors. Overall, patients with MEN1 have a decreased life expectancy as surgical cure is unlikely given their predisposition to multiple and recurrent tumors. Historically, death occurred relatively early in life and was attributed to the complications of a gastrinoma, insulinoma, or severe hyperparathyroidism ${ }^{[2,3]}$. Advancements in medical treatment have enabled MEN1 patients to live longer with patients now rarely dying from said complications ${ }^{[3]}$. Patients with MEN1, particularly those with non-functioning NETs, now require long-term monitoring for late disease manifestations.

\subsection{Thymic neuroendocrine tumors}

Thymic NETs are extremely rare, accounting for only $0.4 \%$ of the 3,692 anterior mediastinal neoplasms reported to the Surveillance, Epidemiology, and End Results (SEER) database between the years of 1973-2006 ${ }^{[4]}$. Risk factors for developing a thymic NET include MEN1 syndrome, male gender, history of tobacco use, and Caucasian race ${ }^{[2,4]}$. Although thymic NETs may develop in roughly 3\%-8\% of MEN1 patients, this special population accounts for $25 \%$ of all reported cases ${ }^{[2,5]}$. When associated with MEN1, these tumors are typically non-functional and reported as late complications of the disease. One third of patients are reported to be asymptomatic on presentation and diagnosed after incidental findings on imaging ${ }^{[6]}$. If symptoms are present, they are more likely secondary to local compression leading to hoarseness, cough, dyspnea, or chest pain. Thymic NETs are associated with a poor long-term prognosis secondary to their highly aggressive nature, late detection, poor response to therapy, and high rates of recurrence.

Due to the rarity of thymic NETs, most of what is known about them comes from case reports and several retrospective studies. While the importance of detecting these tumors in patients with MEN1 is recognized given their aggressive behavior, there are currently no universal screening guidelines. Some experts suggest screening male patients with MEN1 with a chest CT every 3 years, starting at age $25^{[5]}$, while others suggest a yearly chest CT in all patients with MEN1 older than age $25^{[2]}$. Despite these recommendations, screening remains controversial as early detection has not been shown to 
improve prognosis. Surgical resection of thymic NETs is recommended, but the role of chemotherapy or radiation therapy has not yet been clearly defined ${ }^{[2]}$. Some authors recommend prophylactic thymectomy at the time of parathyroidectomy, but this practice does not have proven benefit ${ }^{[1,2]}$.

\subsection{Pancreatic neuroendocrine tumors}

Pancreatic NETs are also very rare, making up $1 \%-3 \%$ of all pancreatic neoplasms ${ }^{[1,7,8]}$. These are the primary life-threatening manifestation of MEN1. Approximately 65\% of pancreatic NETs are non-functional with no associated clinical syndrome or biochemical markers, thus posing a difficult diagnostic challenge for the clinician ${ }^{[1,7]}$. Magnetic resonance imaging (MRI), CT, and SRS are imaging modalities commonly used to identify pancreatic NETs. Endoscopic ultrasound (EUS) is typically used to localize and stage these tumors; EUS also allows for confirmation of the diagnosis via fine needle aspiration ${ }^{[7]}$. The sensitivity of EUS is operator dependent and reported to be $87 \%$ in pancreatic NETs less than $2 \mathrm{~cm}$ in size ${ }^{[7]}$.

Functioning pancreatic NETs may be detected by the imaging modalities described above or by measuring biochemical markers specific to the tumor, i.e. insulin (insulinoma), gastrin (gastrinomas), glucagon (glucagonomas), and serotonin (small bowel NET). Chromogranin A (CgA) is another biomarker that has been studied extensively and found to be expressed by normal and malignant neuroendocrine cells ${ }^{[9,10]}$. The function of CgA is not completely understood. Day-to-day fluctuations in CgA levels of up to $25 \%$ are seen in both healthy patients and those with NETs, along with up to a three-fold increase after a meal ${ }^{[9]}$. CgA is considered a sensitive but non-specific biomarker in the detection of NETs. False positive results may occur in patients with renal failure, chronic gastritis, other forms of cancer, or in patients with prolonged exposure to proton pump inhibitors ${ }^{[8,9]}$. Values also vary based upon the assay, with abnormal levels typically defined as two to three times the upper limit of normal of the assay being used. The highest levels ( $>150$ times the upper limit of normal) have been reported in the ileal and gastroenteropancreatic NETs associated with MEN1 syndrome. ${ }^{[9]}$ Some authors caution that poorly differentiated tumors ${ }^{[9]}$ and insulinomas ${ }^{[11]}$ do not cause CgA levels to rise and may therefore be missed. Despite these limitations, CgA is still considered the best available biomarker for detecting NETs and assessing tumor burden, disease progression, and response to therapy ${ }^{[9-11]}$.

As with thymic NETs, there is no consensus regarding detection and surveillance of pancreatic NETs in MEN1 patients. It is generally acknowledged that surveillance is important given the morbidity and mortality associated with these tumors. Currently, a widely accepted MEN1 surveillance protocol ${ }^{[1]}$ recommends annual measurements of fasting gastrin, insulin, glucose, CgA, glucagon, vasointestinal polypeptide and pancreatic polypeptide, along with radiographic screening of the pancreas and duodenum with CT, MRI, or EUS at least annually. These guidelines also recommend close surveillance for pituitary and parathyroid lesions through annual measurement of plasma calcium, parathyroid hormone, prolactin and insulin-like growth factor 1 (IGF-1) and CT or MRI imaging of the pituitary and adrenal glands every 3 years. The aforementioned guideline also recommends CT or MRI of the chest every 1-2 years to detect thymic and bronchopulmonary neuroendocrine tumors.

\subsection{Case conclusion}

Mr. P.B. has no clinical evidence of a pancreatic NET or recurrence of a thymic NET to date. Imaging studies (chest CT, SRS and EUS) have all been negative. His CgA level was not measured prior to thymectomy. His only abnormal biomarkers include an elevated CgA level (56 ng/ml, reference range 1.9-15 ng/ml) and glucagon $(201 \mathrm{pg} / \mathrm{ml}$, reference < $134 \mathrm{pg} / \mathrm{ml}$ ). The increase in CgA is currently unexplained but may be secondary to his twice daily use of a PPI. The increase in glucagon may be secondary to his diabetes.

\section{References}

[1] Thakker RV, Newey PJ, Walls GV, et al. Endocrine Society. Clinical practice guidelines for multiple endocrine neoplasia type 1 (MEN1). J Clin Endocrinol Metab. 2012 Sep; 97(9): 2990-3011. PMID: 22723327 http://dx.doi.org/10.1210/jc.2012-1230

Published by Sciedu Press 
[2] Gibril F, Chen YJ, Schrump DS, et al. Prospective study of thymic carcinoids in patients with multiple endocrine neoplasia type 1. J Clin Endocrinol Metab. 2003 Mar; 88(3): 1066-81. PMID: 12629087

[3] Ito $\mathrm{T}$, Igarashi $\mathrm{H}$, Uehara $\mathrm{H}$, et al. Causes of death and prognostic factors in multiple endocrine neoplasia type I: A prospective Study. Comparison of 106 MEN1/Zollinger-Ellison syndrome patients with 1613 literature MEN1 patients with or without pancreatic endocrine tumors. Medicine (Baltimore). 2013 May; 92(3): 135-81. PMID: 23645327 http://dx.doi.org/10.1097/MD.0b013e3182954af1

[4] Gaur P, Leary C, Yao JC. Thymic neuroendocrine tumors: A SEER database analysis of 160 patients. Ann Surg. 2010 Jun; $251(6)$ : 1117-21. PMID: 20485130 http://dx.doi.org/10.1097/SLA.0b013e3181dd4ec4

[5] Teh BT, McArdle J, Chan SP, et al. Clinicopathologic studies of thymic carcinoids in multiple endocrine neoplasia type 1. Medicine. 1997 Jan; 76(1):21. PMID: 9064485

[6] Chaer, R, Massad MG, Evans A, et al. Primary neuroendocrine tumors of the thymus. Ann Thorac Surg. 2002 Nov; 74(5): $1733-40$. PMID: 12440652

[7] Nozari N, Nikfam S, Nikmanesh A, et al. Clinical and pathological features of non-functional neuroendocrine tumors of pancreas: a report from Iran. Middle East J Dig Dis. 2014 Jul; 6(3):151-5. PMID: 25093063

[8] Paik WH, Ryu JK, Song BJ, et al. Clinical usefulness of plasma chromogranin a in pancreatic neuroendocrine neoplasm. J Korean Med Sci. 2013 May; 28(5): 750-4. PMID: 23678268 http://dx.doi.org/10.3346/jkms.2013.28.5.750

[9] Modlin IM, Gustafsson BI, Moss SF, et al. Chromogranin A- biological function and clinical utility in neuro endocrine tumor disease. Ann Surg Oncol. 2010 Sep; 17(9): 2427-43. PMID: 20217257 http://dx.doi.org/10.1245/s10434-010-1006-3

[10] Nikou GC, Marinou K, Thomakos P, et al. Chromogranin A levels in diagnosis, treatment, and follow-up of 42 patients with non-functioning pancreatic endocrine tumors. Pancreatology. 2008; 8(4-5): 510-9. PMID:18765956 http://dx.doi.org/10.1159/000152000

[11] Qiao XW, Qiu L, Chen YJ, et al. Chromogranin A is a reliable serum diagnostic biomarker for pancreatic neuroendocrine tumors but not for insulinomas. BMC Endocr Disord. 2014 Aug; 14: 64. PMID: 25099181 http://dx.doi.org/10.1186/1472-6823-14-64 